\title{
Effect of extinction on the high-energy optical response of photonic crystals
}

\author{
L. A. Dorado and R. A. Depine* \\ Grupo de Electromagnetismo Aplicado, Departamento de Física, Facultad de Ciencias Exactas y Naturales, \\ Universidad de Buenos Aires, Buenos Aires, Argentina \\ H. Míguez \\ Instituto de Ciencia de Materiales de Sevilla, Consejo Superior de Investigaciones Científicas, Sevilla, Spain
} (Received 20 February 2007; published 7 June 2007)

\begin{abstract}
An analysis of the optical response of photonic crystals in the high-order band energy range is herein presented. High and abruptly fluctuating specular reflectance is predicted for perfect lattices at those energies even in the absence of any photonic gap or pseudogap. As optical extinction is gradually introduced, it is possible to reproduce experimental results found in the literature and which have recently been the subject of an intense debate. Band structure calculations demonstrate that extinction is extraordinarily amplified in the high-energy range and is responsible for the features so far observed in that range in real crystals.
\end{abstract}

DOI: 10.1103/PhysRevB.75.241101

The large spatial anisotropy of the dielectric constant in two- and three-dimensional photonic crystals (PCs) results in very complex band structures. ${ }^{1}$ In the higher-order bands, the interaction of multiple wave vectors propagating along different crystalline directions gives rise to very low dispersion modes, and although full gaps may open if the dielectric contrast is high enough, most practically feasible lattices present a wide passband in which the photon density of states fluctuates abruptly. ${ }^{2}$ Interesting fundamental phenomena with great potential applications have been observed when light propagates through higher band modes, such as the superprism effect ${ }^{3}$ or beam self-focusing. ${ }^{4}$ Different experimental and theoretical works have deepened our knowledge of this energy range. ${ }^{5-7}$ However, the optical features observed in the reflectance and transmittance spectra of real crystals are not yet fully understood.

Here we present a complete description of the optical response of real PCs in the high-energy range, in which the spatial variation of the dielectric constant is on the order of the wavelength. We chose a face-centered cubic (fcc) lattice of low-refractive-index spheres to analyze the effect of extinction on the optical properties in this range. We start by describing the band structure and predicting the optical response of a fcc crystal with almost no losses and then gradually introduce extinction in its components. At those energies for which very low-dispersion propagation modes are attained, we predict that perfect lattices should present a strongly fluctuating optical response that rapidly smooths out as extinction is gradually increased. A similar behavior has been reported by Modinos et al. ${ }^{8}$ although it seems to have been overlooked in the literature so far. Furthermore, we observe that a residual imaginary part of the dielectric constant introduced in the spheres gives rise to extraordinarily high values of the extinction for the high-energy region. We found that this extinction amplification effect is responsible for the shape of all actual reflectance and transmittance spectra so far reported, all experimental data available being explained by our model.

Transmittance and reflectance spectra, as well as the band structure, were calculated using the code reported by Stefanou et $a l \cdot{ }^{9,10}$ and based on the Korringa-Kohn-Rostoker (KKR) wave calculation method. To study the convergence
PACS number(s): 42.70.Qs, 41.20.Jb, 78.20.Bh, 78.40.-q

of the fields, the values of the code parameters that control the number of terms in both the spherical-wave and planewave expansions of the electric field, have been increased from $L M A X D=7$ and IGD $=21$ to $L M A X D=19$ and IGD $=99$, respectively. For the calculations shown in this paper, we used 41 two-dimensional (2D) reciprocal lattice vectors in the plane-wave expansions and spherical waves with angular momentum up to $l_{\max }=7$. These are the minimum values providing good convergence in that range. Lower values of $l_{\max }$ and fewer reciprocal lattice vectors still give a correct result for lower-energy bands, enough to describe the optical response around the stop band, although not sufficient to provide trustworthy information on the region we are interested in.The model structure employed is a close-packed fcc lattice of spheres of dielectric constant $\varepsilon_{s}=2.4964+\varepsilon_{i}$ in a medium of $\varepsilon=1$. This value corresponds to latex spheres in air, a structure that has been repeatedly fabricated and typically considered of high structural quality in the field of 3D PCs. ${ }^{11}$ The largest accessible surface is determined by the crystal growth direction, typically the [111]. ${ }^{12}$ We will focus on the optical properties when light impinges in that particular direction since they are best known.

The calculated band structure along the $\Gamma L$ direction for a sphere of dielectric constant $\varepsilon_{s}=2.4964+0.0001 i$, along with the reflectance spectrum in a selected range are shown in Fig. 1. Energy is expressed in reduced units $a / \lambda$ where $a$ is the lattice constant. The band structure of this lattice hardly differs from that calculated using a purely real $\varepsilon_{s}$, at least in the energy range we are interested in, since the imaginary part of the wave vector is negligible. Interestingly, a similar approach has been employed to calculate the scattered electron spectra of semiconductors. ${ }^{13}$ Although the KKR method converges for $\varepsilon_{i}=0$, the introduction of this residual extinction allows one to obtain the reflectance in a range in which its fluctuations are so abrupt in the lattice without losses that its analysis is almost unaffordable. In Fig. 1(c), the reflectance for a crystal made of 21 layers is shown. It can be observed that a strongly fluctuating reflectance is expected for lowdielectric-contrast fcc lattices in which extinction can be neglected, for energies above the pseudogap. Reflectance may reach very high values in the absence of any gap, while total transmission may occur for some other narrow spectral 

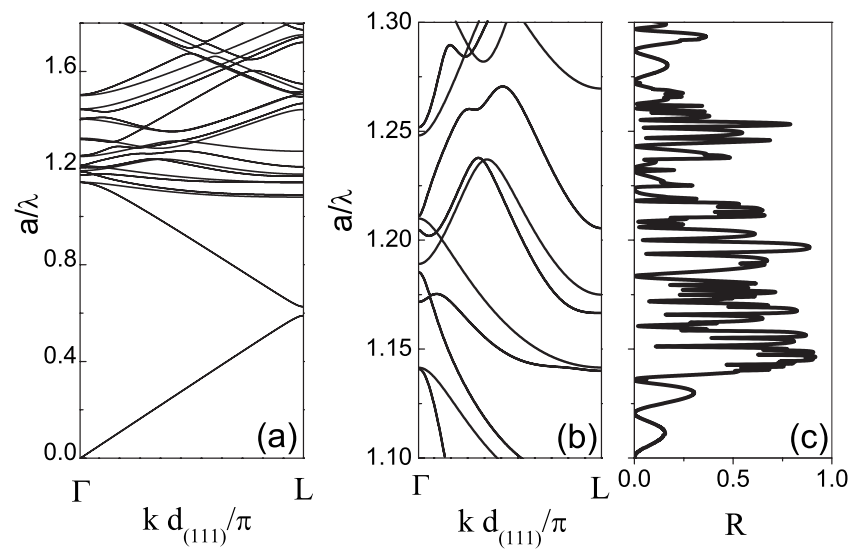

FIG. 1. (a) Band structure along the $\Gamma L$ direction of a perfect fcc crystal consisting of spheres of dielectric constant $\varepsilon_{s}=2.4964$ in air. (b) Zoom of the band structure in the range $1.1 \leqslant a / \lambda \leqslant 1.3$. (c) Specularly reflected light spectrum for $\varepsilon_{s}=2.4964+0.0001 i$.

ranges. Our results reproduce the abrupt changes in the specular reflectance already predicted in Ref. 8 for perfect lattices without absorption. None of the fcc structures reported so far have shown any of the features shown in Fig. 1 for photon energies $a / \lambda>1$, but much smoother ones. The reasons are addressed in what follows.

Real structures present imperfections ${ }^{14,15}$ that cause light to be scattered diffusely, removing part of the energy from the specularly reflected or forward-transmitted beams. This extinction is here modeled by adding a small imaginary part in the $\varepsilon$ of the spheres of our crystal, which accounts for all possible sources of losses in the lattice.

The relation between the optical response and the band structure of a sphere lattice having optical extinction was analyzed. The real $\operatorname{Re}(k)$ and imaginary $\operatorname{Im}(k)$ parts of the wave vector along the $\Gamma L$ direction are plotted separately in Figs. 2(a) and 2(b) for $\varepsilon_{s}=2.4964+0.04 i$, that is, $\varepsilon_{i}$ two orders of magnitude larger than that used in Fig. 1. As expected, in the low-energy region $(a / \lambda \leqslant 0.9)$, the effect of

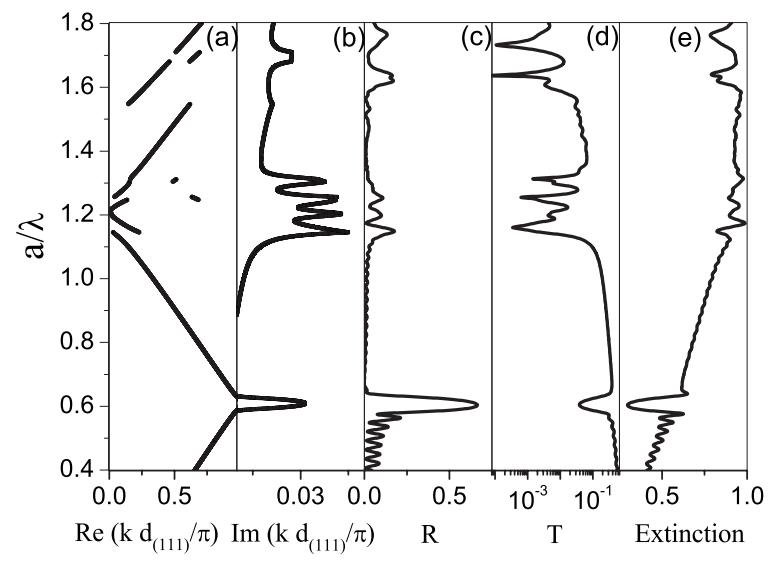

FIG. 2. Band structure, reflectance, transmittance, and extinction spectra of a fcc crystal consisting of spheres of dielectric constant $\varepsilon_{s}=2.4964+0.04 i$ in air. Real (a) and imaginary (b) parts of the wave vector along the $\Gamma L$ direction. Specular reflectance (c), forward transmittance (d), and extinction (e) spectra.
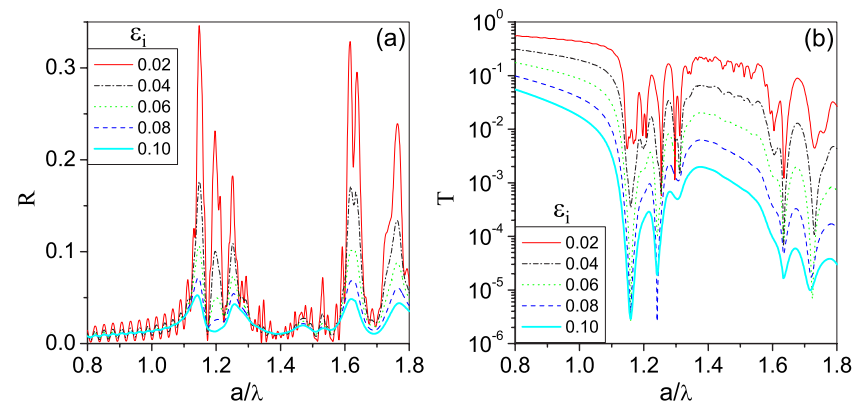

FIG. 3. (Color online) (a), (b) Specularly reflected and forwardtransmitted light spectra for spheres of dielectric constant $\varepsilon_{s}$ $=2.4964+i \varepsilon_{i}$, and for several values of $\varepsilon_{i}$, indicated in the insets of the figure.

such losses is weak, although it has to be taken into account if a precise description of the measurements is needed. ${ }^{16,17}$ For $a / \lambda \leqslant 0.9$, the band dispersion is almost identical to that of the crystal without extinction: the wave vector may be considered real and linear with frequency, $\operatorname{Im}(k)$ becoming significantly larger within the pseudogap. However, well above it, the band structure becomes completely different from that of a crystal without losses (see Fig. 1). In the range $1.1 \leqslant a / \lambda \leqslant 1.3, \operatorname{Im}(k)$ reaches up to four clearly distinguishable maxima whose positions precisely coincide with the minima observed in the forward transmittance, Fig. 2(d), and with three of the maxima of the reflectance spectrum, Fig. 2(c). This coincidence reveals that the features of the experimental optical spectra in the high-energy region are due to the excitation of light modes with a complex wave vector in a finite-thickness PC slab. For these modes, extinction, estimated from the requirement of energy conservation, is greatly amplified, as shown in Fig. 2(e). This amplification effect may be put into practice to enhance the efficiency of devices in which large photon absorption is required in very short distances, such as solar cells or photodetectors. Our results confirm that the optical properties of a PC at wavelengths similar to or shorter than the lattice parameter $(a / \lambda$ $\geqslant 1$ ) are much more sensitive to any modification of the structure, in good agreement with previous studies. ${ }^{18}$ The extinction we are introducing has little effect on the optical properties at lower band energies, but gives rise to huge distortion of both the spectra and the band structure at higher frequencies. Similarly, any small structural modification can give rise to large variations of the optical response at higher frequencies. A similar absorption amplification effect has previously been described for photonic crystal waveguides, in which it was found that loss scales inversely with group velocity. ${ }^{19,20}$

The parameter $\varepsilon_{i}$ was varied in order to analyze the effect of different amounts of structural disorder. Figures 3(a) and 3(b) show the specularly reflected and the forwardtransmitted spectra for 21 sphere layers, a value used in Ref. 5 for real fcc crystals. For a very low $\varepsilon_{i}$, two sets of reflectance peaks (deep transmission dips) are attained for $1.1 \leqslant a / \lambda \leqslant 1.3$ and $1.55 \leqslant a / \lambda \leqslant 1.8$. As we increase $\varepsilon_{i}$, the reflectance peaks decrease, while the dips in transmission become less pronounced.

The comparison between the calculations and experimen- 

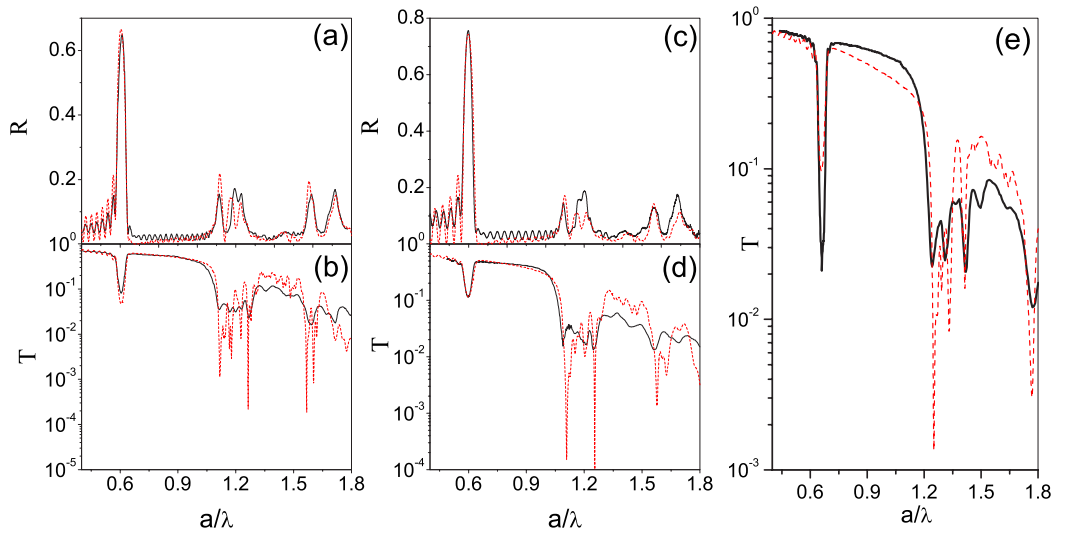

FIG. 4. (Color online) Measured reflectance and transmittance spectra extracted from Ref. 5 (a), (b) and Ref. 20 (c), (d) (solid lines) and the calculated spectra $\left(\varepsilon_{i}=0.04\right.$, dashed lines $)$ for spheres of refractive index 1.59 in air. (e) Measured transmittance spectrum extracted from Ref. 21 (solid line) and the calculated spectrum $\left(\varepsilon_{i}\right.$ $=0.02$, dashed line) for spheres of refractive index 1.42 in air. tal spectra extracted from the literature ${ }^{5,21,22}$ are shown in Fig. 4. The best fit to experimental data was attained for $\varepsilon_{i}$ around 0.04 and adding some dispersion in the real part of the dielectric constant, which accounts for a small refractive index increase as we move toward higher energies. Excellent agreement with experiment is found for the number of peaks in reflectance and dips in transmittance, their position and intensity in the two high-energy ranges of interest (1.1 $\leqslant a / \lambda \leqslant 1.3$ and $1.55 \leqslant a / \lambda \leqslant 1.8)$. These ranges are different in the case of the spectra shown in Fig. 4(e), since they correspond to silica $(n=1.42)$ spheres in which the higherorder band effects occur at higher energies. All these lattices have been built by evaporation-induced self-assembly, and their optical response is fitted using much lower values of extinction than needed for other lattices (results not shown here) grown using other methods, ${ }^{11,23}$ thus probing their higher optical quality. The reflectance spectra of the PC slabs with the highest losses, Fig. 3(a), present just two peaks of low intensity in the range $1.1 \leqslant a / \lambda \leqslant 1.3$, instead of a fine three-peak structure. This trait coincides with what has repeatedly been observed for colloidal crystals which are known to present a higher density of defects. However, none of the experimental data so far reported presents such intense features as those shown in Fig. 1, which indicates that there is still a long way to go to achieve true defect-free fcc crystals. These results prove that the rich optical properties at higher band energies are very sensitive to the presence of defects and may be used to assess the quality of the lattice.

Our results demonstrate that, in the high-energy region, perfect 3D PCs should present a very intense and highly fluctuating reflectance spectrum, which smooths out due to extinction sources of different sort. All measurements reported up to date at those frequencies for sphere fcc lattices show just a peak or dip structure reminiscent of the welldefined one expected for a perfect lattice. The agreement between our model and the optical experiments supports the idea that extinction plays a crucial role in determining the shape and intensity of these features. However, full understanding of the physical origin of the properties of perfect lattices at those frequencies is still a challenge. Deficient coupling to flat dispersion bands ${ }^{18}$ or the reminiscence of gaps due to the anticrossing of bands ${ }^{20}$ have been proposed as causes of this very complex high-energy spectrum. Lack of coupling due to the existence of blind modes, not excitable for symmetry reasons, has been previously discarded. ${ }^{24}$
Recently, the abrupt drop of intensity occurring at $a / \lambda \sim 1$ has been attributed to bands that scatter light in nonforward directions, as explained in Ref. 6. Therein, the authors point out that diffraction is responsible for the dips observed in the transmission spectra of sphere fcc lattices. In order to shed some light on this debate, we calculated the spectral distribution of the energy diffracted modes for a fcc lattice both deposited on a substrate of index of refraction $n_{2}=1.53$ (as in Ref. 6) and self-standing $\left(n_{2}=1\right)$. In both cases, the incoming medium has $n_{1}=1$. The ratio of the power carried by each diffracted mode to the incident power is $R_{m, l}$ (reflected) or $T_{m, l}$ (transmitted). The integers $(m, l)$ give the order of diffraction, $(m, l)=(0,0)$ being the specularly reflected or forward-transmitted light, which are typically measured.

Figure 5 shows the spectrum of $T_{0,0}$ and that of $\sum_{m \neq 0, l \neq 0} T_{m, l}$ (the sum of the intensities of all diffracted modes in the homogeneous transmission medium) for both the glass-supported lattice and the self-standing one. The parameters of the structure are similar to those used in Fig. 2. Our calculations show that, when the presence of a substrate $\left(n_{2}=1.53\right)$ is considered, diffraction in the glass starts for $a / \lambda \sim 1.1$, as observed in Ref. 6 . This diffraction coincides with the beginning of the drop in transmission observed experimentally and reproduced by the calculation of $T_{0,0}$ shown in Fig. 5(a). When the response of the self-standing crystal is analyzed $\left(n_{2}=1\right)$, an abrupt change in transmittance is still attained for $a / \lambda \sim 1$ even when no diffraction occurs in that range, as shown in Figs. 5(c) and 5(d). In fact, diffraction in air starts (that is, $\sum_{m \neq 0, l \neq 0} T_{m, l} \neq 0$ ) at $a / \lambda \sim 1.63$, as expected from the condition of conservation of the tangential component of the wave vectors for a close-packed 2D sphere lattice:

$$
\frac{a}{\lambda} \geqslant \frac{\sqrt{2}}{n_{2}} \sqrt{l^{2}+\frac{(2 m+l)^{2}}{3}} .
$$

This expression explains the onset of diffracted modes only within the substrate $\left(n_{2}=1.53\right)$ and not in the incoming medium at $a / \lambda \sim 1.1$ for the supported lattice. These results show that scattering in nonforward directions is not responsible for the main transmission dips observed at $a / \lambda \sim 1.1$, as proposed in Ref. 6. The fact that the drop in transmittance occurs at the same spectral value as the diffracted modes in Ref. 6 is just a coincidence originating in the value of the refractive index of the substrate. The two phenomena are not related, as proved in Figs. 5(c) and 5(d). Diffracted modes 

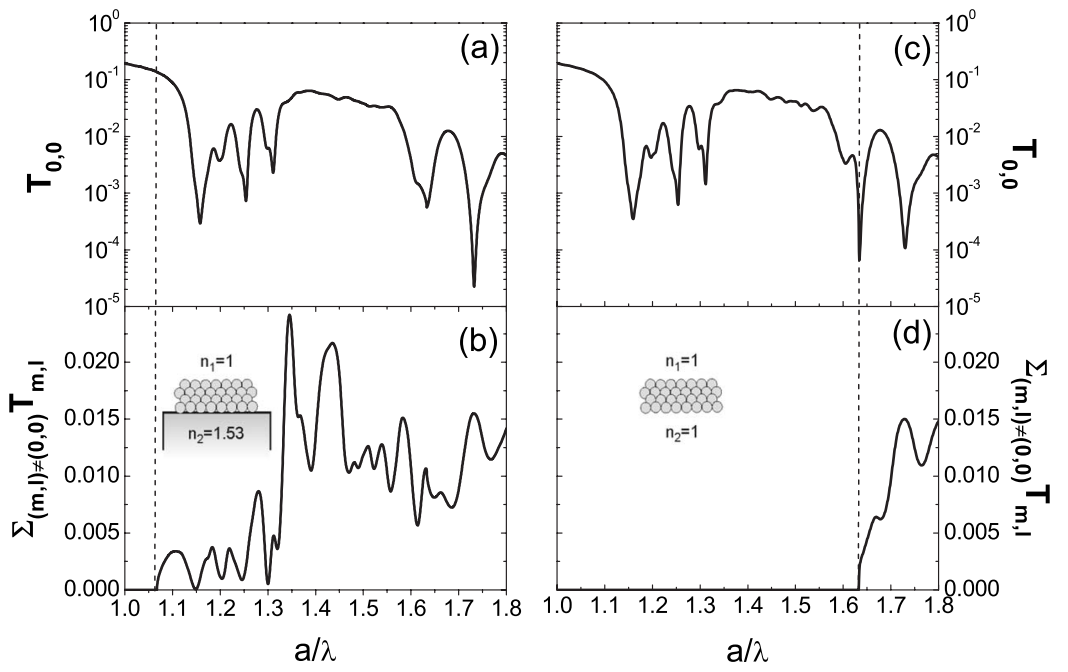

FIG. 5. Forward transmittance $T_{0,0}$ and total transmitted diffracted light, $\Sigma_{m, l \neq 0} T_{m, l}$, spectra for the glass-supported lattice (a), (b) and for the self-standing one (c), (d). Vertical dashed lines indicate the onset of diffraction expected for each type of substrate according to Eq. (1). within the substrate provoke a further although not significant decrease of the transmittance of sustained lattices at frequencies that will depend on the refractive index of the substrate. Thus, we take into account all possible sources of scattering and attain good agreement with all previously reported measurements, even in spectral regions well above the fundamental stop band where diffraction effects come into play. Previous theoretical studies have not provided simultaneous satisfactory fitting of experimental reflectance and transmittance as we do herein.

In conclusion, we have realized an integral approach to the optical response of 3D PCs in the higher-order band energy range. We have demonstrated that extinction due to intrinsic defects determines the shape of the experimental spectra that have been so far reported. Calculations of the crystal photon modes, also performed considering extinction, shows a clear correlation between the behavior of the imaginary part of the wave vector and the optical features observed in that range. Our results imply that the higher-order band region of PCs may be used to amplify losses in devices in which enhanced absorption is desirable, such as solar cells or photon detectors.

This work has been realized in the framework of a joint Spanish-Argentinian cooperation project CSIC-CONICET (Grant No. 2005AR0070). R.A.D. and L.D. acknowledge support from Consejo Nacional de Investigaciones Científicas y Técnicas (CONICET), Universidad de Buenos Aires (UBA), and Agencia Nacional de Promoción Científica y Tecnológica (ANPCYT-BID 802/OC-AR03-14099). H.M. acknowledges financial support from the Ramón Areces Foundation and the Spanish Ministry of Science and Education under Grant No. MAT2005-03028.
*Electronic address: rdep@df.uba.ar

${ }^{1}$ K. Sakoda, Optical Properties of Photonic Crystals (SpringerVerlag, Berlin, 2001).

${ }^{2}$ K. Busch and S. John, Phys. Rev. E 58, 3896 (1998).

${ }^{3}$ H. Kosaka, T. Kawashima, A. Tomita, M. Notomi, T. Tamamura, T. Sato, and S. Kawakami, Phys. Rev. B 58, R10096 (1998).

${ }^{4}$ A. Martínez, H. Míguez, A. Griol, and J. Martí, Phys. Rev. B 69, 165119 (2004).

${ }^{5}$ J. F. Galisteo-López and C. López, Phys. Rev. B 70, 035108 (2004).

${ }^{6}$ F. García-Santamaría, J. F. Galisteo-López, P. V. Braun, and C. López, Phys. Rev. B 71, 195112 (2005).

${ }^{7}$ A. Balestreri, L. C. Andreani, and M. Agio, Phys. Rev. E 74, 036603 (2006).

${ }^{8}$ A. Modinos, N. Stefanou, and V. Yannopapas, Opt. Express 8, 197 (2001).

${ }^{9}$ N. Stefanou, V. Karathanos, and A. Modinos, J. Phys.: Condens. Matter 4, 7389 (1992).

${ }^{10}$ N. Stefanou, V. Yannopapas, and A. Modinos, Comput. Phys. Commun. 113, 4977 (1998); 132, 189 (2000).

${ }^{11}$ M. Allard, E. Sargent, E. Kumacheva, and O. Kalinina, Opt. Quantum Electron. 34, 27 (2002).

${ }^{12}$ P. Jiang, J. F. Bertone, K. S. Hwang, and V. L. Colvin, Chem. Mater. 11, 2132 (1999).
${ }^{13}$ J. B. Pendry, Low Energy Electron Diffraction (Academic Press, London, 1974).

${ }^{14}$ Yu. A. Vlasov, V. N. Astratov, A. V. Baryshev, A. A. Kaplyanskii, O. Z. Karimov, and M. F. Limonov, Phys. Rev. E 61, 5784 (2000).

${ }^{15}$ A. F. Koenderink, A. Lagendijk, and W. L. Vos, Phys. Rev. B 72, 153102 (2005).

${ }^{16}$ J. F. Galisteo-López and W. L. Vos, Phys. Rev. E 66, 036616 (2002).

${ }^{17}$ J. F. Galisteo-López, M. Galli, M. Patrini, A. Balestreri, L. C. Andreani, and C. López, Phys. Rev. B 73, 125103 (2006).

${ }^{18}$ K. Busch and S. John, Phys. Rev. Lett. 83, 967 (1999).

${ }^{19}$ D. Gerace and L. C. Andreani, Opt. Lett. 29, 1897 (2004).

${ }^{20}$ S. Hughes, L. Ramunno, J. F. Young, and J. E. Sipe, Phys. Rev. Lett. 94, 033903 (2005).

${ }^{21}$ H. Míguez, V. Kitaev, and G. Ozin, Appl. Phys. Lett. 84, 1239 (2004).

${ }^{22}$ S. Wong, V. Kitaev, and G. A. Ozin, J. Am. Chem. Soc. 125, 15589 (2003).

${ }^{23}$ R. Mayoral, J. Requena, J. S. Moya, C. López, A. Cintas, H. Míguez, F. Meseguer, L. Vazquez, M. Holgado, and A. Blanco, Adv. Mater. (Weinheim, Ger.) 9, 257 (1997).

${ }^{24}$ F. López-Tejeira, T. Ochiai, K. Sakoda, and J. Sánchez-Dehesa, Phys. Rev. B 65, 195110 (2002). 